\title{
Tohoku-Hiroshima-Nagoya planetary spectra library: a method for characterizing planets in the visible to near infrared ${ }^{\star}$
}

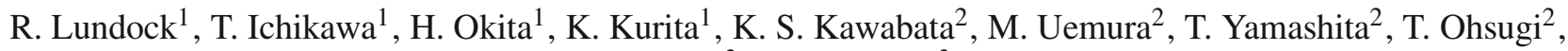 \\ S. Sato $^{3}$, and M. Kino ${ }^{3}$ \\ 1 Astronomical Institute, Tohoku University, Aoba, Sendai 980-8578, Japan \\ e-mail: rlundock@astr.tohoku.ac.jp \\ 2 Astrophysical Science Center, Hiroshima University, Kagamiyama 1-3-1, Higashi-Hiroshima 739-8526, Japan \\ 3 Department of Physics, Nagoya University, Furo-cho, Chikusa-ku, Nagoya 464-8602, Japan
}

Received 27 May 2009 / Accepted 28 August 2009

\section{ABSTRACT}

\begin{abstract}
Context. A comprehensive framework for comparing spectral data from different planets has yet to be established. This framework is needed for the study of extrasolar planets and objects within the solar system.

Aims. We completed observations to compile a library of planet spectra for all planets, some moons, and some dwarf planets in the solar system to study their general spectroscopic and photometric natures.

Methods. During May and November of 2008, we acquired spectra for the planets using TRISPEC, which is capable of simultaneous three-band spectroscopy across a wide wavelength range of $0.45-2.5 \mu \mathrm{m}$ with low resolving power $(\lambda / \Delta \lambda \sim 140-360)$.

Results. Patterns emerge when comparing the spectra. By analyzing their general spectroscopic and photometric natures, we show that it is possible to distinguish between gas planets, soil planets, and ice planets. These methods can be applied to extrasolar observations acquired using low resolution spectrography or broad-band filters.

Conclusions. The present planet spectral library is the first library to contain observational spectra for all of the solar system planets, based on simultaneous observations at visible and near infrared wavelengths. This library will be a useful reference for analyzing extrasolar planet spectra and calibrating planetary data sets.
\end{abstract}

Key words. astronomical data bases: miscellaneous - planets and satellites: general

\section{Introduction}

Successive improvements to telescopes and probe missions have provided an unprecedented amount of information about the planets orbiting the Sun and other stars. The numbers of known planets and bodies that are "not quite planets" increase every year. Ceres, Pluto and Eris were reclassified as dwarf planets in 2006. Haumea and Makemake were subsequently added to the dwarf planet category (WGPSN 2009). It is an open question whether small brown dwarves can be differentiated from large gas giants. Because of the enormous amount of data taken so far by many authors, we know a great deal about the nature of each planet in the solar system. However, the framework for discussing "planets in general" has lagged behind.

Spectra exist for all of the solar system's planets and many of its smaller objects. To find spectral libraries in many cases it is necessary to return to the 1970's and early 1980's when the first quality spectra of planets were being obtained. Particularly prolific studies during this era were, e.g., McCord \& Westphal (1971), Fink et al. (1976), McCord et al. (1979), Fink \& Larson (1979), Clark (1980, 1981), and McCord (1981). However, this was also a time of rapid technological development and even within a single research group, observational techniques changed. Between their 1976 and 1979 observations, the Fink group at Kitt Peak upgraded from a 90 inch telescope to a $4 \mathrm{~m}$

$\star$ THN Planet spectra library is only available in electronic form at the CDS via anonymous ftp to

cdsarc.u-strasbg.fr $(130.79 .128 .5)$ or via

http://cdsweb.u-strasbg.fr/cgi-bin/qcat?]/A+A/507/1649 telescope (Fink et al. 1976; Fink \& Larson 1979). The Pollack group using the Kuiper Airborne Observatory used an uncooled, circular variable-filter wheel for their 1975 observations of Venus and Jupiter (Pollack et al. 1978a). They upgraded to a cooled filter wheel for their 1976 observations of the Galilean satellites (Pollack et al. 1978b). Few observations have been performed for spectral libraries in the past two decades. Examples of spectral data at a broad range of wavelengths are Earth's spectra from 0.7 to $2.4 \mu \mathrm{m}$ (Turnbull et al. 2006) and the IRTF spectral library (Rayner et al. 2008), which includes four planets (Jupiter, Saturn, Uranus, and Neptune) observed by SpeX in the near-infrared $(0.8-5.5 \mu \mathrm{m})$

Most data for the spectral libraries were taken using a unique combination of telescope and instrument. The resolution, responses, and wavelength coverage differed for each data set. It would be difficult to combine published spectral data at various wavelengths, because the data were taken for different phase and/or rotation of the planets. Few data simultaneously obtained across a wide wavelength range from optical to infrared, are available.

In the present study, we show the results of simultaneous spectroscopic observations at wavelengths from optical to infrared for all planets, some moons, and some dwarf planets in the solar system to study their general spectroscopic and photometric natures. Accounts of the observations and data reduction are presented in Sects. 2 and 3, respectively. The spectra of all targets that we observed are compared in Sect. 4 along with the broad-band colors obtained by integrating the spectra. The colors of other objects in the solar system, which are available in 
the literature, are compared with our results. In Sect. 5, we discuss our results and relevant literature in conjunction with the classification of gas, soil, and ice planets.

\section{Observations}

All observations for the present compilation of the spectral library were performed at Higashi-Hiroshima Observatory using the $1.5 \mathrm{~m}$ Kanata telescope and TRISPEC (Triple Range Imager and SPECtrometer with Polarimetry), a simultaneous optical and near-infrared imager, spectrograph, and polarimeter (Watanabe et al. 2005). Two dichroic mirrors split the incoming light from the telescope into three beams, one optical channel $(0.45-0.90 \mu \mathrm{m})$ and two infrared channels IR1 $(0.90-1.85 \mu \mathrm{m})$ and IR2 $(1.85-2.5 \mu \mathrm{m})$. The instrument is capable of simultaneous three-band spectroscopy across a wavelength range of $0.45-2.5 \mu \mathrm{m}$. We selected the slit of $300 \mu \mathrm{m}$ width, which gives the resolving power $(R=\lambda / \Delta \lambda)$ of $R=138,142$, and 360 for optical, IR1, and IR2, respectively. The TRISPEC slit length is $28 \mathrm{~mm}$, which subtends 7 arcmin on the sky.

The observations were carried out during May 2-13, and November $19-27$ of 2008 . While the overall data quality is good, shorter than $0.47 \mu \mathrm{m}$ and between 0.9 and $1.0 \mu \mathrm{m}$ the spectra are not reliable because of the dichroic coating problem with the beam splitters. There is the possibility of thermal contamination from Earth's atmosphere and the telescope beyond $2.4 \mu \mathrm{m}$. Around 1.4 and $1.8 \mu \mathrm{m}$, Earth's atmosphere has strong water absorption bands, where the signal quality is low.

The bodies observed were: Mercury, Venus, Earth, Luna, Mars, Ceres, Jupiter, Io, Europa, Ganymede, Callisto, Saturn, Dione, Rhea, Titan, Uranus, Neptune, Pluto, and Saturn's ring (Table 1). We obtained Earth's spectra by observing earthshine, the light from the Earth which is reflected by the dark side of the moon. We followed the procedure of Woolf et al. (2002) for acquiring and analyzing earthshine. In May, we acquired a Lunar spectrum corresponding to a bright crater, while the bulk of the surface (mare) was observed in November.

The log of the observations is listed in Table 1 along with the brightness in $V$ band, the date, and the phase angle (PhA) defined by the Sun, target, and observer at the time of the observation. Earthshine shows us an integrated spectra of the entire Earth. Therefore, phase angle is the angle formed by the Sun: Earth: Luna. Standard stars for the correction of atmospheric absorption and the spectral response of TRISPEC were observed just before and after observation of each target. The repeat observations provide us with information about sky background variation during observations. The standard star, its spectral type, and $V$ magnitude for each target are listed in the last three columns.

Special care was taken in the observations for large objects, bright objects, and Saturn and its ring.

Large targets: the angular sizes of Jupiter and Saturn are larger than the 4.5 arcsec angle subtended by the spectrograph slit. To acquire spectra of these planets, we aligned the slit along a north/south line through the center of planet; each planet's axis of rotation is at a slight angle to the slit. These spectra do not include light from the entire planet as the spectrum for an object smaller than the slit width would; only light from the part visible through the slit is sampled. We use these spectra as first approximations to the reflection spectra of unresolved gas giants, and note that the scatter in the multiple observations of Saturn would probably decrease if we were able to sample more of its surface. The spectra for sky subtraction were obtained from the sky adjacent to the target.
For earthshine observations, the lunar disk fills the entire spectrograph slit, so that we had to take dedicated sky background frames to the east and west of Luna. These frames were taken using the same exposure times and procedures as observations of the dark portions of Luna. The illuminated portion of Luna is bright enough for sky glow not to contribute noticeably, and can be safely neglected (Turnbull et al. 2006). We took sky background frames to the east and west. Our background frames were placed 30 arcmin both in front of and behind Luna's center point. Our earthshine observation was made at 10 arcmin from the center on the dark side and our observations for Luna were made at 10 arcmin on the illuminated side. Thus, we had equal spacing of 20 arcmin between the exposure points. This allowed us to account for light from the bright surface that had been scattered 20 degrees and would thus show up in our dark side exposure.

Bright targets: for bright targets such as Jupiter and crescent Luna, detector saturation became a problem. We partially closed the telescope dome slit to reduce the light gathering power of the telescope. We used this technique only with bright objects, so that thermal noise from the dome did not have a noticeable effect on the signal quality even near $2.5 \mu \mathrm{m}$.

Saturn and its ring: because of the north/south orientation of the slit, most of the signals shown for Saturn include contamination from the planet's strong ring system. We expect that the ring spectrum and gas planets spectrum do not resemble each other. Therefore, we obtained a spectrum for Saturn without its ring during the November observations, by rotating the spectrograph slit by 90 degrees and placing it on Saturn's north pole. As stated above, we are aware that sampling a small part of the surface does not necessarily reflect the integrated spectrum of the planet, but we will use the resultant spectrum as an approximation.

\section{Data analysis}

We followed the standard procedure for flat fielding, sky subtraction, and spectrum extraction, using IRAF. To correct for sky absorption and the spectral response of TRISPEC, we observed standard stars with airmasses similar to the target. Because the exposure time used for each of TRISPEC's three channels has an effect on the true exposure time of the other two channels, we were careful to use exactly the same exposure times for the standard as for the target. After the spectra had been extracted and wavelength calibrated, we divided each planet spectrum by the observed spectrum of the corresponding standard star, then multiplied by the calculated true spectrum of the star's spectral type taken from the ISAAC database of simulated spectral-type spectra (Pickels 1998). The standard stars that we used for each object and their spectral types are listed in Table 1.

For moons, there is the special problem of light from the parent planet. The amount of scattered light in either the telluric atmosphere or the telescope is a function of radius from the planet. Thus, lines of equal light pollution form rings around the planet. During data analysis, we learned by experience that, when we observe a moon close to the parent planet, the straight line of the spectrograph slit does not offer a good approximation to the rings of equal light pollution. Even though we subtracted the adjacent sky signal, this step was not sufficient to completely remove the scattered light from the planet. When observing moons, we should align the spectrograph slit along the moon's orbit plane. In this way, the sky used for sky subtraction will include areas that have more and less light pollution than the sky in front of the target, so that we should take an average to determine the 
Table 1. Observation Log.

\begin{tabular}{|c|c|c|c|c|c|c|}
\hline 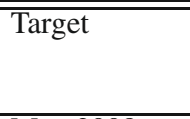 & $\overline{\text { Date }}$ & $\begin{array}{l}\text { Phase } \\
\text { angle } \\
\text { (deg) }\end{array}$ & $\begin{array}{c}V \\
\text { (mag) }\end{array}$ & $\begin{array}{c}\text { Standard } \\
\text { star }^{\dagger}\end{array}$ & $\begin{array}{c}\text { Spectral } \\
\text { type }\end{array}$ & $\begin{array}{c}V \\
(\mathrm{mag}) \\
\end{array}$ \\
\hline \multicolumn{7}{|l|}{ May 2008} \\
\hline Callisto & $05 / 05 / 08$ & 10 & 6.3 & BS7264 & F2II & 2.89 \\
\hline Saturn & $05 / 05 / 08$ & 6 & 1.0 & BS4101 & A0 & 2.89 \\
\hline Dione & 05/05/08 & 6 & 10.4 & BS4101 & A0 & 2.89 \\
\hline Titan & $05 /(05,06) / 08$ & 6 & 8.4 & BS4101 & A0 & 2.89 \\
\hline Io & $05 / 06 / 08$ & 10 & 5.4 & BS7264 & F2II & 2.89 \\
\hline Saturn's Ring & 05/06/08 & 6 & 1.0 & BS4101 & A0 & 2.89 \\
\hline Rhea & 05/06/08 & 6 & 9.7 & BS4101 & A0 & 2.89 \\
\hline Jupiter & 05/08/08 & 10 & -2.4 & BS7264 & F2II & 2.89 \\
\hline Europa & 05/07/08 & 10 & 5.6 & BS7264 & F2II & 2.89 \\
\hline Neptune & 05/07/08 & 2 & 7.9 & HIP105946 & B9III/IV & 7.09 \\
\hline Uranus & 05/(07, 11)/08 & 2 & 5.9 & BS8911 & A0p & 4.49 \\
\hline Mercury & 05/11/08 & 96 & 0.0 & BS1641 & B3V & 3.17 \\
\hline Earth & $05 / 11 / 08$ & 81,84 & -2.5 & BS2890 & $\mathrm{A} 2 \mathrm{~V}$ & 1.58 \\
\hline Luna (crater) & $05 / 11 / 08$ & 97 & -9.8 & BS2890 & $\mathrm{A} 2 \mathrm{~V}$ & 1.58 \\
\hline Pluto & $05 / 11 / 08$ & 1 & 15 & HIP87881 & $\mathrm{A} 0 \mathrm{~V}$ & 10.08 \\
\hline Mars & $05 / 12 / 08$ & 35 & 1.3 & BS2890 & A2V & 1.58 \\
\hline \multicolumn{7}{|l|}{ Nov. 2008} \\
\hline Saturn's Ring & $11 / 19 / 08$ & 6 & 1.2 & BS4101 & A0 & 2.89 \\
\hline Saturn & $11 /(19,22) / 08$ & 6 & 1.2 & BS4101 & A0 & 2.89 \\
\hline Neptune & $11 /(20,25,26) / 08$ & 2 & 7.9 & HIP104167 & A0 & 10.10 \\
\hline Venus & $11 /(20,26) / 08$ & 63,65 & -4.2 & BS7264 & F2II & 2.89 \\
\hline Uranus & $11 / 20 / 08$ & 3 & 5.8 & HIP112880 & A0 & 8.22 \\
\hline Earth & $11 / 21 / 08$ & 70 & -2.5 & HIP50793 & A2 & 9.13 \\
\hline Luna (mare) & $11 / 21 / 08$ & 108,109 & -9.3 & BS4101 & A0 & 2.89 \\
\hline Titan & $11 / 25 / 08$ & 6 & 8.6 & HIP50793 & A2 & 9.13 \\
\hline Ceres & $11 / 25 / 08$ & 23 & 8.4 & HIP50793 & A0 & 9.13 \\
\hline Rhea & $11 / 25 / 08$ & 6 & 9.9 & HIP50793 & A2 & 9.13 \\
\hline Dione & $11 / 25 / 08$ & 6 & 10.6 & HIP50793 & A2 & 9.13 \\
\hline Io & $11 / 26 / 08$ & 8 & 5.8 & HIP106831 & B9V & 8.27 \\
\hline Ganymede & $11 / 26 / 08$ & 8 & 5.4 & HIP106831 & B9V & 8.27 \\
\hline
\end{tabular}

$\dagger$ BS and HIP refer to UKIRT (2008) and Perryman et al. (1997), respectively.

true amount of scattered light in the telluric atmosphere in front of the target.

In analyzing spectra of the solar system planets, authors studying a particular planet's reflectivity often divide the planet spectra by the Sun's spectrum. Groups focusing on a single feature will instead normalize with respect to a continuum. Because there is no consensus on which method to use, we use the spectra without applying either method in the following discussion. The earthshine data requires more procedures than the other observations. Because the light that we see as earthshine is reflected by Luna, the spectrum is affected by the wavelength dependence of Luna's reflectivity. Therefore, we divided the earthshine spectra by a spectrum of moonlight obtained on the same night, with corrections for phase angle (Woolf et al. 2002). Most earthshine research groups publish their results in terms of Earth's reflectivity; we have taken the additional step of multiplying by the Sun's spectrum to compare Earth's spectrum to the remainder of the planets.

\section{Results}

All spectra that we analyzed are shown in Fig. 1 and Appendix A. The spectra are normalized at $0.7 \mu \mathrm{m}$. In this paper, we use the term "planet" loosely, to include moons and rings as well. Several objects were observed a multiple number of times as listed in Table 1. These multiple observations are plotted separately in Fig. 1, because spectra depend on the object's rotation, the changes in phase angle, or the slit location on extended objects. For example, the spectrum of Mars shows dramatic changes based on the part of the surface visible, and individual sections of Mars show time-dependent changes (McCord \& Westphal 1971). We also show the spectrum of the Sun for comparison.

\subsection{Classification by spectral features}

To highlight the patterns that emerge when comparing the spectra, we classify the planets into gas, soil, and ice from their spectral characteristics with the following criteria. There are also some anomalous spectra which cannot be easily attributed to one of the categories.

Gas planets - Jupiter, Saturn, Uranus, Neptune, and Titan: the spectra of gas planets are dominated by strong methane absorption around 1.0, 1.17, 1.7, and $2.2 \mu \mathrm{m}$ (Strong et al. 1993). We specifically avoid the term "gas giant" because Titan's spectra match the others so well. The atmospheres of Jupiter, Saturn, Uranus, and Neptune consist primarily of hydrogen and helium. The bulk of Titan's atmosphere is nitrogen. Small amounts of methane are sufficient to cause deep, broad absorption features. Changes in the shapes of these features indicate the concentration of methane.

Soil planets - Mercury, Earth, Luna, Mars, Ceres, Io, and Ganymede: Mars is a typical example of the category that we call soil planets. Compared to the gas planets, soil planets have a 


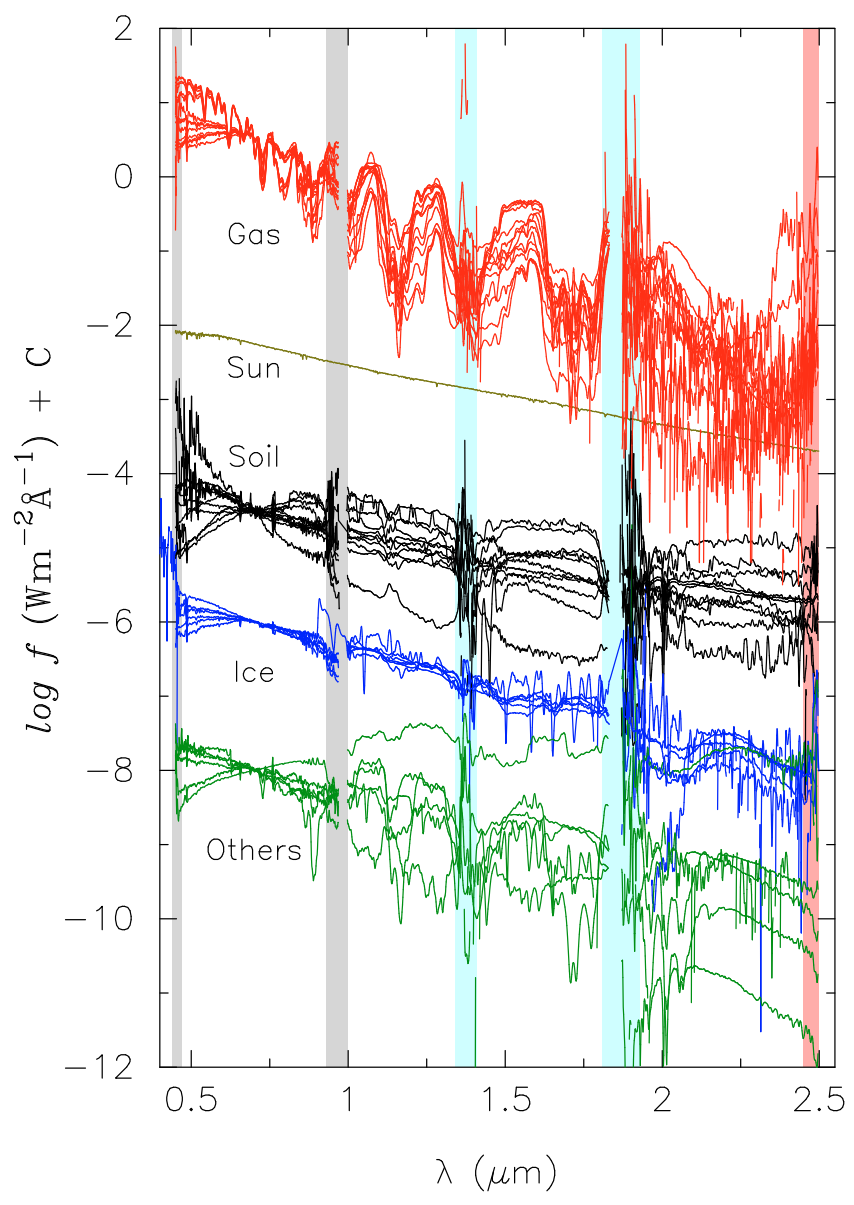

Fig. 1. All spectra of solar system objects taken by TRISPEC. Each spectrum is normalized at $0.7 \mu \mathrm{m}$. Flux, $f$, is arbitrarily offset so as to group the objects into gas, soil, ice, and anomalous others. The Sun spectrum is taken from the STScI archive (http://www.stsci.edu/ hst/observatory/cdbs/calspec.html).

simple shape. The flux remains roughly constant across the optical and near infrared. One exception is the Luna crater, which exhibits a steep spectrum in visible light (see Sect. 5 for more details).

Ice planets - Saturn's Ring, Dione, Europa, Rhea, and Pluto: the group is characterized by broad and deep absorption of crystalline water ice centered near $2.05 \mu \mathrm{m}$, which is roughly $0.3 \mu \mathrm{m}$ wide. The steep blue slope and absorption lines of ice planets match the spectra of water ice in the laboratory (Clark 1981; Grundy et al. 1999). Pluto's spectrum has a slight wave caused by its methane ice composition (Protopapa et al. 2008), but its overall shape is very similar to that of water ice.

Others: we place the four objects with contaminated spectra into the Others class of objects. They are the May observations for Callisto, Io, and Rhea, and the November observation of Dione. For all objects except Rhea, we were also able to obtain an uncontaminated spectrum.

\subsection{Color-color distribution}

One inherent disadvantage of spectroscopy is that it requires long observation exposure times. For dim objects and surveys of a large number of objects color observations are more practical. From our spectral data, we calculate the colors of the planets. The magnitudes are defined by the flux integrated with filter responses of $V\left(\lambda_{\mathrm{eff}}=0.55 \mu \mathrm{m}\right)$ (Johnson \& Morgan 1953),

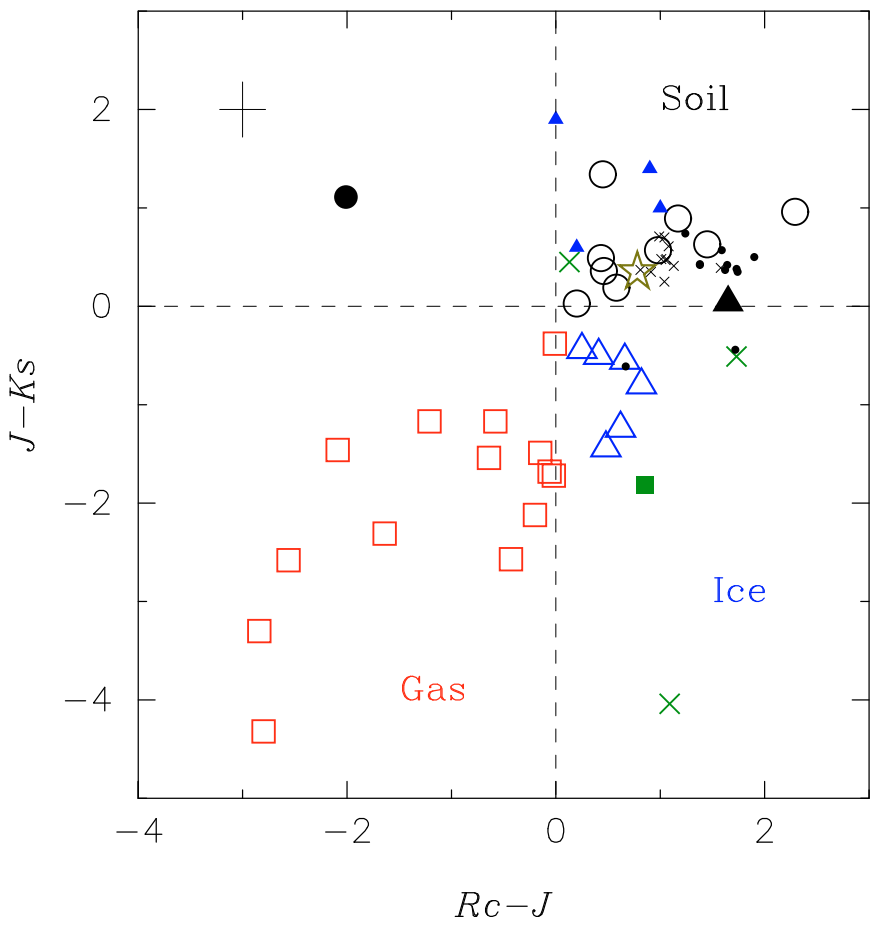

Fig. 2. Color-color diagram for the objects listed in Table 2. The magnitudes are obtained by integrating the spectra. Circle, triangle, square, and cross symbols show the classes of soil, ice, gas, and anomalous others (see Table 2). A filled circle, filled squares and a filled large triangle represent the Luna crater, Venus, and Ganymede, respectively. Small dots show Kuiper belt objects (Delsanti et al. 2004). Small crosses are trans neptunian objects and centaurs (DeMeo et al. 2009). Filled small triangles are comet Halley 1986 III (Gehrz \& Hey 1992). The solar color is shown by a star. The typical error bars of our observations are depicted at the upper left corner.

$R c(0.66 \mu \mathrm{m}), I c(0.81 \mu \mathrm{m})$ (Cousins 1978), and $J(1.25 \mu \mathrm{m})$, $H(1.64 \mu \mathrm{m})$, and $K \mathrm{~s}(2.15 \mu \mathrm{m})$ (Tokunaga et al. 2002). Colors are defined so as to be 0.0 for all colors of Vega ${ }^{1}$. The colors of Jupiter, Saturn, and Neptune taken with SpeX (Rayner et al. 2008) were obtained in the same manner. The results of the color analysis are shown in Table 2. The repeated observations of the standard stars before and after that of a target allow us to estimate the errors. The resultant errors in optical and infrared colors are $\Delta(V-R c)=0.13, \Delta(R-I c)=0.09, \Delta(R c-J)=0.22$, $\Delta(J-K \mathrm{~s})=0.28$, and $\Delta(H-K \mathrm{~s})=0.30$. The errors mainly originated in variable sky background during a target's observations. The larger errors in infrared should be caused by variations in the water vapor in the telluric atmosphere. In Fig. 2, we plot the $J-K$ versus $R c-J$ colors. Again, in Fig. 2 and Table 2, multiple observations of the same target are shown. Some of the scatter is the result of photometeric error, but we cannot exclude the possibility that there are also time-dependent changes in the target. For example, an extended photometric time series in $J$ and $K$ bands of Neptune exhibited rapid color variation within a day by $\sim 3 \mathrm{mag}$ in $(J-K)$ because of the planet's rotation (Belton et al. 1981).

We plot additional color information for Kuiper belt objects (KBO) (Delsanti et al. 2004), transneptunian objects (TNO), centaurs (DeMeo et al. 2009), and comet Halley 1986 III (Gehrz \& Hey 1992), for which $R c, J$, and $K$ data are available.

\footnotetext{
1 The spectrum of Vega is taken from http: //www . stsci . edu/hst/ observatory/cdbs/calspec.html
} 
Table 2. Photometric colors of targets.

\begin{tabular}{|c|c|c|c|c|c|c|c|}
\hline$\overline{\text { Object }}$ & Class & $\overline{\bar{V} V-R}$ & 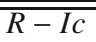 & 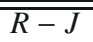 & $\bar{~} J-K$ & $\overline{H-K \mathrm{~s}}$ & Remark \\
\hline The Sun & & 0.38 & 0.35 & 0.78 & 0.35 & 0.05 & (1) \\
\hline \multicolumn{8}{|l|}{ May 2008} \\
\hline Earth $\left(\mathrm{PhA}=81^{\circ}\right)$ & Soil & 0.09 & 0.02 & 0.43 & 0.49 & 0.56 & desert \& ocean \\
\hline Earth $\left(\mathrm{PhA}=84^{\circ}\right)$ & Soil & 0.13 & 0.15 & 0.20 & 0.03 & 0.21 & desert \& ocean \\
\hline Luna (crater) & Soil & -0.88 & -0.81 & -2.01 & 1.11 & 1.27 & descending (2) \\
\hline Mercury & Soil & 1.17 & 1.17 & 2.29 & 0.96 & 0.37 & \\
\hline Mars & Soil & 1.03 & 0.90 & 1.17 & 0.89 & 0.37 & \\
\hline Pluto & Ice & 0.35 & 0.28 & 0.41 & -0.50 & -0.82 & \\
\hline Dione & Ice & 0.12 & 0.08 & 0.25 & -0.44 & -0.23 & \\
\hline Europa & Ice & 0.69 & 0.49 & 0.82 & -0.80 & -0.53 & \\
\hline Saturn's Ring & Ice & 0.46 & 0.41 & 0.62 & -1.24 & -0.89 & (2) \\
\hline Jupiter & Gas & 0.28 & 0.06 & -0.64 & -1.54 & -1.33 & (2) \\
\hline Saturn & Gas & 0.18 & -0.12 & -0.20 & -2.12 & -2.31 & $\mathrm{w} /$ ring (2) \\
\hline Titan $(05 / 05)$ & Gas & 0.37 & 0.01 & -0.58 & -1.17 & -0.30 & \\
\hline Titan $(05 / 06)$ & Gas & 0.67 & 0.33 & -0.01 & -0.38 & -0.10 & \\
\hline Uranus $(05 / 11)$ & Gas & -0.28 & -1.01 & -2.84 & -3.30 & -3.31 & \\
\hline Neptune & Gas & -0.17 & -0.78 & -1.64 & -2.31 & -2.14 & \\
\hline Callisto & - & 0.25 & -0.17 & 1.09 & -4.04 & -3.95 & \\
\hline Io & - & 0.81 & 0.36 & 1.73 & -0.51 & -0.26 & \\
\hline Rhea & - & 0.98 & 1.04 & 3.68 & 1.09 & 0.76 & \\
\hline \multicolumn{8}{|l|}{ Nov. 2008} \\
\hline Earth & Soil & 0.08 & 0.00 & 0.58 & 0.19 & -0.06 & ocean \\
\hline Luna (mare) $\left(\mathrm{PhA}=108^{\circ}\right)$ & Soil & 0.39 & 0.43 & 0.98 & 0.57 & 0.03 & ascending (2) \\
\hline Luna (mare) $\left(\mathrm{PhA}=110^{\circ}\right)$ & Soil & 0.52 & 0.32 & 0.45 & 1.34 & 0.81 & ascending (2) \\
\hline Ceres & Soil & 0.19 & 0.24 & 0.46 & 0.36 & 0.06 & \\
\hline Ganymede & Soil & 1.04 & 0.87 & 1.65 & 0.04 & -0.08 & \\
\hline Io & Soil & 0.62 & 0.71 & 1.45 & 0.63 & -0.10 & \\
\hline Rhea & Ice & 0.29 & 0.17 & 0.48 & -1.44 & -1.26 & \\
\hline Ring & Ice & 0.54 & 0.31 & 0.66 & -0.55 & -0.43 & (2) \\
\hline Saturn (11/19) & Gas & 0.48 & 0.12 & -0.06 & -1.68 & -1.79 & $\mathrm{w} /$ ring $(2)$ \\
\hline Saturn((11/19) & Gas & 0.54 & 0.13 & -0.02 & -1.72 & -1.85 & w/o ring, N.pole(2) \\
\hline Saturn $(11 / 22)$ & Gas & 0.26 & 0.03 & -0.15 & -1.49 & -1.49 & $\mathrm{w} / \operatorname{ring}(2)$ \\
\hline Titan & Gas & 0.55 & 0.06 & -0.43 & -2.57 & -2.20 & \\
\hline Uranus & Gas & -0.25 & -0.87 & -2.80 & -4.32 & -4.20 & \\
\hline Neptune $(11 / 20)$ & Gas & -0.32 & -0.81 & -2.56 & -2.58 & -2.55 & \\
\hline Neptune $(11 / 25)$ & Gas & -0.32 & -0.78 & -2.09 & -1.46 & -1.53 & \\
\hline Neptune $(11 / 26)$ & Gas & -0.12 & -0.73 & -1.21 & -1.17 & -1.14 & \\
\hline Venus $(11 / 20)$ & - & 0.38 & 0.40 & 0.85 & -1.82 & -1.97 & (3) \\
\hline Venus $(11 / 26)$ & - & 0.18 & 0.02 & $-1.70:$ & 2.40: & 1.61: & (3) \\
\hline Dione & - & 0.18 & 0.12 & 0.13 & 0.45 & 0.23 & \\
\hline \multicolumn{8}{|l|}{ SpeX (Rayner et al. 2008) } \\
\hline Jupiter & Gas & & & & -2.75 & -2.55 & \\
\hline Saturn & Gas & & & & -1.78 & -1.96 & \\
\hline Neptune & Gas & & & & -2.63 & -2.58 & \\
\hline
\end{tabular}

(1) The flux data is taken from http://www.stsci.edu/hst/observatory/cdbs/calspec.html

(2) Because the target is larger than the spectrograph slit,these spectra are from part of the instead of the integrated planet spectrum.

(3) The signal in November 20 for Venus is in good agreement with the spectra taken by Pollack (1978). Compared to the Nov. 20th spectrum, the Nov. 26th signal has the same shape, but the region from 1 to $1.8 \mu \mathrm{m}$ appears depressed, though the origin is not known.

\section{Discussion}

In the following, we use the word "blue" to mean that the signal is stronger at shorter wavelengths than at longer wavelengths. In contrast, "red" is used to mean the signal is stronger at longer wavelengths.

The overall slope of the gas planet spectra across the infrared is very blue. The exact shapes of the spectra are determined by the composition of each planet's atmosphere and clouds. A full discussion of the scattering mechanisms of these 5 objects is beyond the scope of this paper. For our purposes, it is sufficient to note that the slope can be modeled by a simple $\lambda^{-4}$ Rayleigh scattering model (Bergstralh \& Neff 1983).

In the visible, the spectra of the gas planets diverge and both Uranus and Neptune are very blue with strong methane absorption lines, while Jupiter, Saturn and Titan have level spectra. Jupiter and Saturn have high clouds which are more reflective in the visible than in the infrared (Fink \& Larson 1979). The reflection/transmission properties of the clouds on Uranus and Neptune are less dependent on wavelength, so that short wavelength light exhibits the same steep blue trend and strong methane absorption as we see in the infrared (Fink \& Larson 1979; Karkoschka 1994). On Titan, aerosols prevent visible light from reaching the same depths in the atmosphere as infrared light (Fink \& Larson 1979; Karkoschka 1994).

Soil planets also exhibit a large dispersion in the optical and infrared spectra. This is not surprising, given the vast differences between the surfaces of Mercury, Earth, and Ceres. Individual spectral features come primarily from silicates, ironbearing minerals, and water in the form of hydrated minerals 
or ice. The Luna crater is the bluest spectrum presented in the soil category of Fig. 1. This spectrum is similar to infrared crater spectra obtained by other researchers (McCord 1981). The spectrum drops sharply below the other soil planets in the visible and stays flat thoughout the infrared. Bright lunar craters contain freshly exposed or young material. The gradual reddening (i.e. the decrease in the reflectance at short wavelengths) is a result of the weathering process of agglutinate accumulation on the surface (Pieters 1986).

In contrast to the spectra of gas and soil planets, the spectra of ice planets vary little with wavelength. While the other two categories have a large dispersion, the individual ice planet spectra closely resemble each other. Even though Pluto consists primarily of methane ice instead of water ice (Protopapa et al. 2008), it is still a close match to the other ice planets.

Although many photometric observations have been performed so far, most were acquired at different epoch in the optical and infrared. Therefore, our color-color diagram (Fig. 2) would reflect a more real nature of the planets than previous studies. In the color-color diagram, the differences between planet types suggested by the individual spectra become obvious. We roughly divide the figure into gas, soil, and ice regions, although some targets are outside these categories.

Soil planets are close to the Sun's color. Ice planets are bluer in $J-K$ than soil planets; gas planets are much bluer than soil planets in both $R c-J$ and $J-K$. Although $R c$-band data is not available, the colors of asteroids in infrared (Baudrand et al. 2001, 2004), which are distributed in the region $J-K>0$ and $I-J>0$, are consistent with the soil class. The location of a blue $\mathrm{KBO}$, (24835) $1995 \mathrm{SM}-55,(R c-J=0.67, J-K=-0.61)$ supports Delsanti's finding indicative of water ice absorption features (Delsanti et al. 2004).

It is interesting to note that even though the Others class of objects were contaminated by stray light from their parent gas planet, in the color-color diagram none of them occupy the gas planet area. The contamination was not strong enough to change the color significantly.

Two topics deserve special attention: Ganymede \& other dirty ice planets and Venus.

Ganymede \& dirty ice: we identified Ganymede as a soil planet because its spectrum has more in common with Mars and Ceres than with the spectra of icy satellites. While Ganymede has a rocky core, the surface is primarily ice. Estimates of the amount of ice on Ganymede's surface have been revised over the years. Current models include dirty ice contaminated with dark particles, probably an iron-rich material (Clark 1980). The dark particles can be dredged up from Ganymede's large rocky core by ice volcanism (Showman et al. 2004). This justifies our classification of Ganymede as soil and shows that there is not a sharp division between soil planets and ice planets. There is a gradual transition through a dirty ice stage as the fractional composition changes.

Comets are well known to be "dirty snowballs". Colors are shown for Halley's comet (Gehrz \& Hey 1992) in Fig. 2. The colors fall in the range of the soil planets, and appear to be slightly redder than soil planets in $J-K$ s color. Laboratory work with mineral grains on water ice frost shows that small amounts of silicate contamination can produce a dramatic change in the near infrared spectra (Clark 1981). In particular there is a dramatic reddening in the wavelengths used to calculate $J-K$ color (Clark 1980).

Venus: Venus does not have a typical soil planet spectrum. This is not unexpected since the solid surface of Venus is hidden beneath a thick, cloud bank. Venus has a $(J-K)$ color that is bluer than any of the soil planets. This color is caused by scattering in Venus's thick atmosphere. Venus does match the spectra for ice planets fairly closely (see Appendix A).

Distinguishing between Venus and ice planets requires being able to differentiate between $\mathrm{H}_{2} \mathrm{O}$ and $\mathrm{CO}_{2}$ absorption features. Venus exhibits strong $\mathrm{CO}_{2}$ lines at $1.4,1.6$, and $2.0 \mu \mathrm{m}$. Water ice has strong lines at $1.5,1.65$ and $2.0 \mu \mathrm{m}$. The ice lines are known to change wavelength based on the physical structure and temperature of the ice (Grundy et al. 1999), making distinguishing between the two types of planets more difficult. The two can be distinguished by their spectra but not by their color. More work, possibly in the visible or ultraviolet, is needed to break the Venus/ice degeneracy. It is possible that Venus represents a fourth class, such as veiled planets, which have a thick atmosphere devoid of the methane features found in gas planets.

\section{Conclusion}

We have acquired spectra for all of the solar system planets and many smaller bodies using TRISPEC on the $1.5 \mathrm{~m}$ Kanata telescope at Higashi-Hiroshima Observatory. The spectra are divided by their characteristic shapes into three distinct groups: gas planets, soil planets, and ice planets. Venus represents a fourth possible group. Our results indicate that low resolution, broad-band spectroscopy across the visible and near infrared can provide a wealth of information about the planets being observed.

In addition to spectroscopy, simple color observations can be used to separate planets into these three categories. Although, Venus and Ganymede demonstrate that the classification system is not perfect, color observations have the advantage that they can be used on fainter and/or more numerous objects than even low resolution spectroscopy.

We originally undertook this research to improve our understanding extrasolar planets. However, even within our own solar system, there are numerous small bodies whose compositions remain unidentified, or unable to be inferred by density calculations that require difficult-to-obtain observations (Grundy et al. 2007). Our work demonstrates the potential for meaningful data in visible and near infrared color surveys of a large number of asteroids, centaurs, and TNO. Our results will provide a useful comparison to the reflection spectra of exoplanets.

The Tohoku-Hiroshima-Nagoya Planet Spectra Library (THN-PSL) presented here is the first library to contain visible and near IR spectra for all of the solar system planets, observed with the same instrument and telescope combination for all planets. This library will be a useful reference for analyzing extrasolar planet spectra, and for comparing between planetary data sets.

Acknowledgements. We thank all members of the TRISPEC development team and the Higashi-Hiroshima Observation team for their hard work which helped to make our observations a success. This work was partially supported by the Mitsubishi Foundation. IRAF is distributed by the National Optical Astronomy Observatories, which are operated by the Association of Universities for Research in Astronomy, Inc., under cooperative agreement with the National Science Foundation.

\section{Appendix A: Complete spectral data}

We present all of our observed spectra. The spectra are normalized at $0.7 \mu \mathrm{m}$. The text data are available upon request from the authors (RL, TI). The complete Tohoku-Hiroshima-Nagoya Planet Spectra Library is also available in text format from the CDS archive. 
R. Lundock et al.: THN-PSL
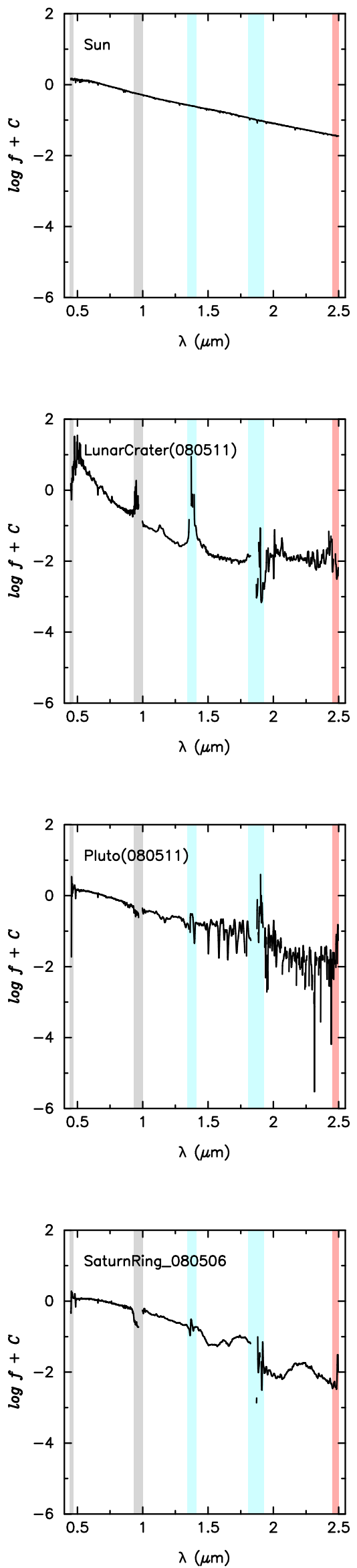
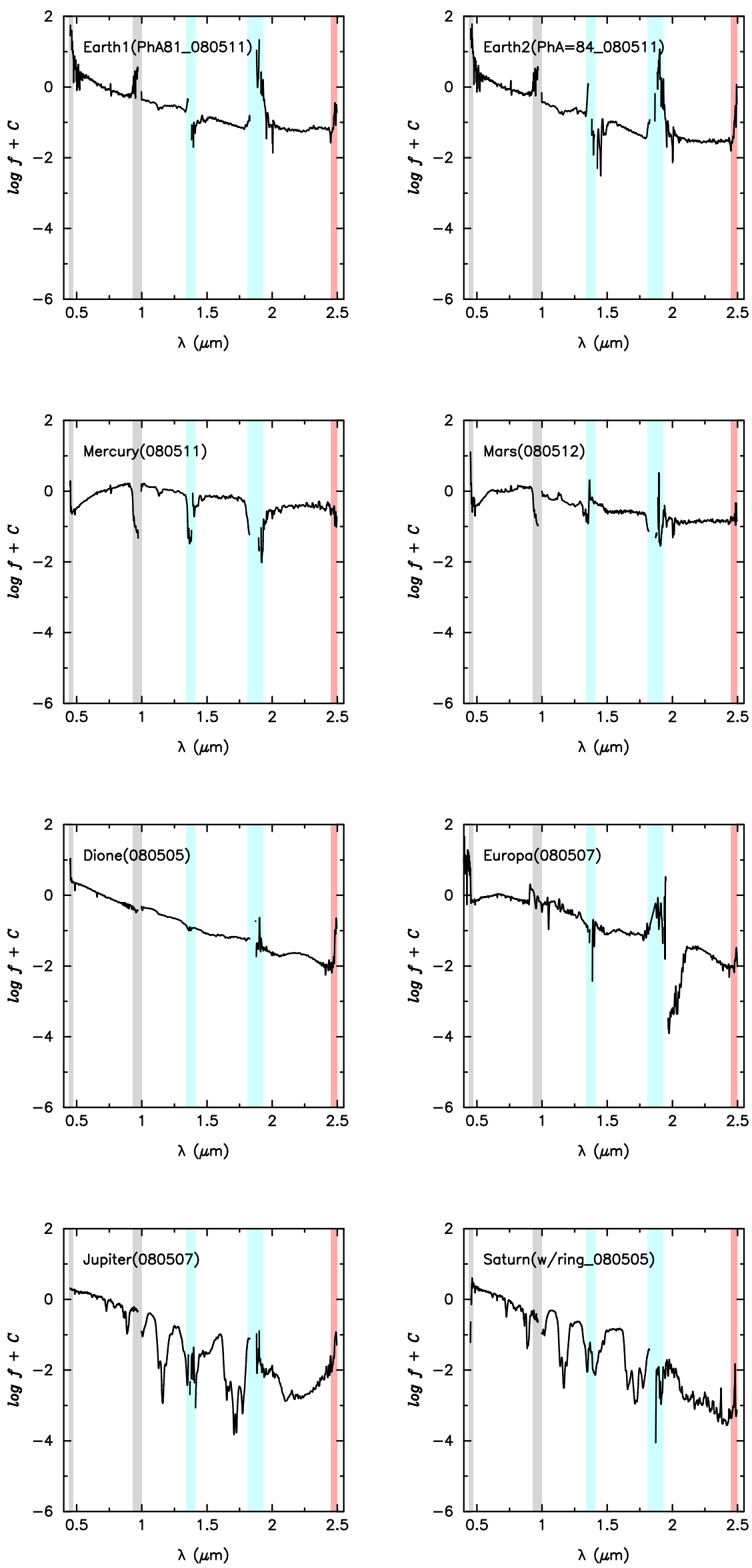

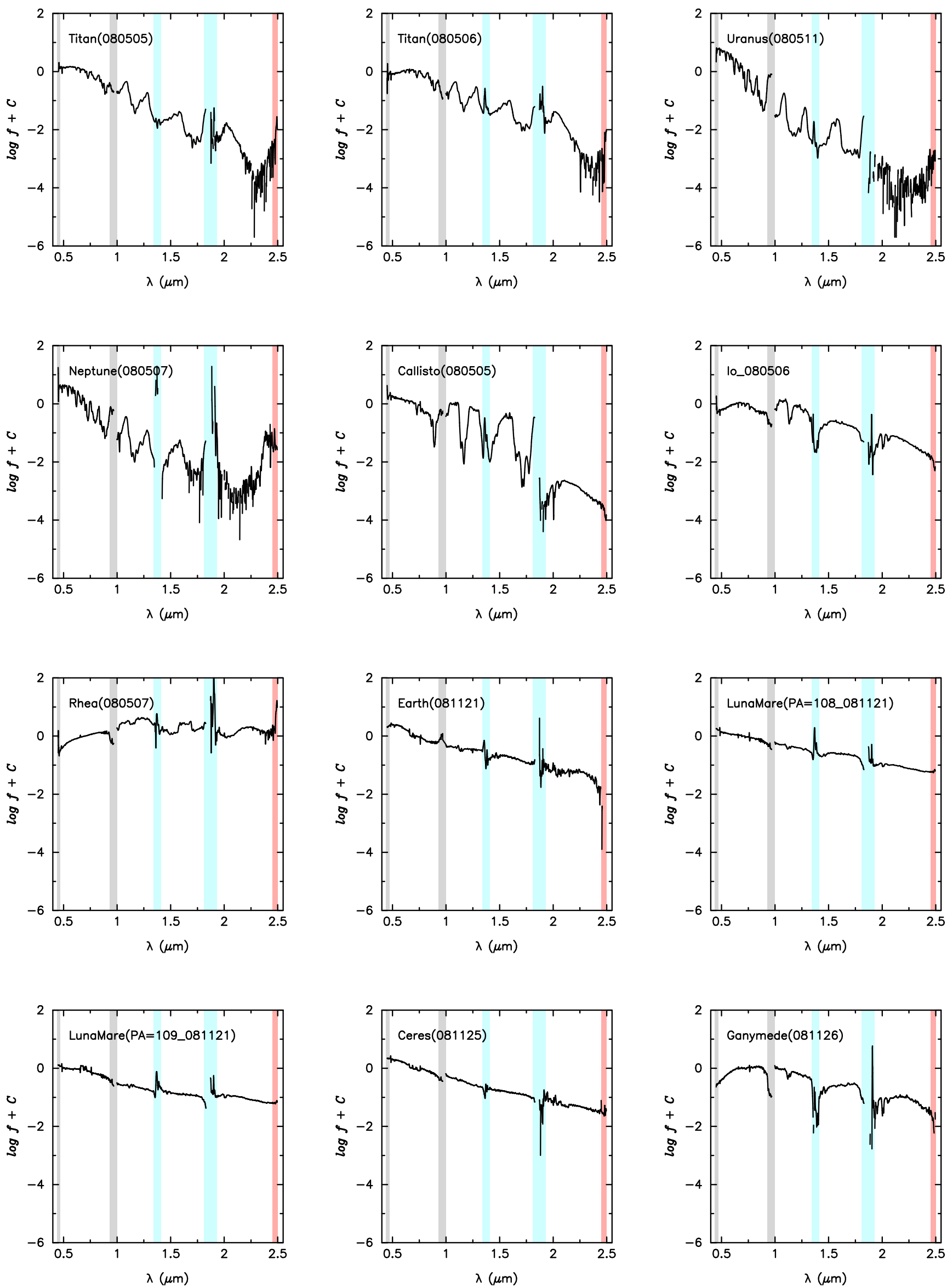
R. Lundock et al.: THN-PSL
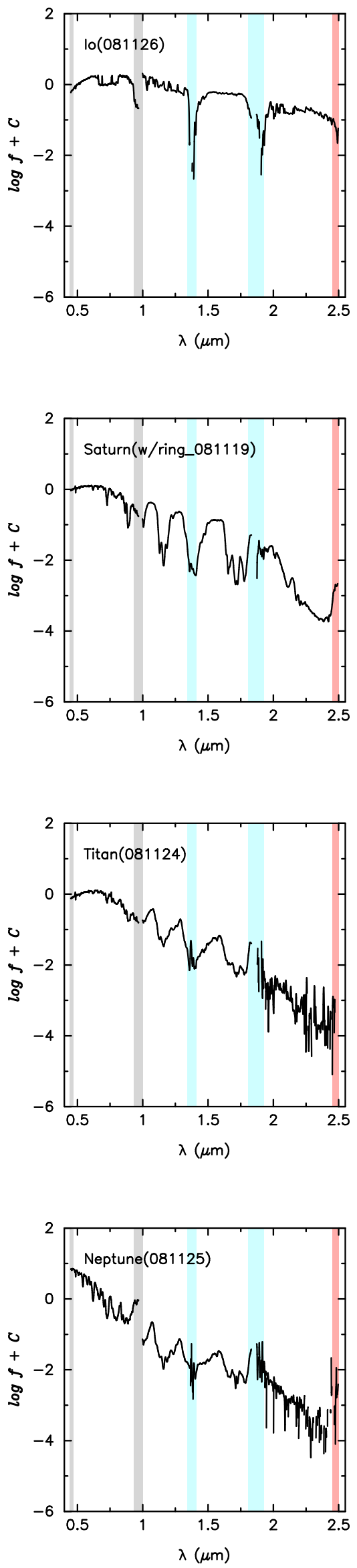
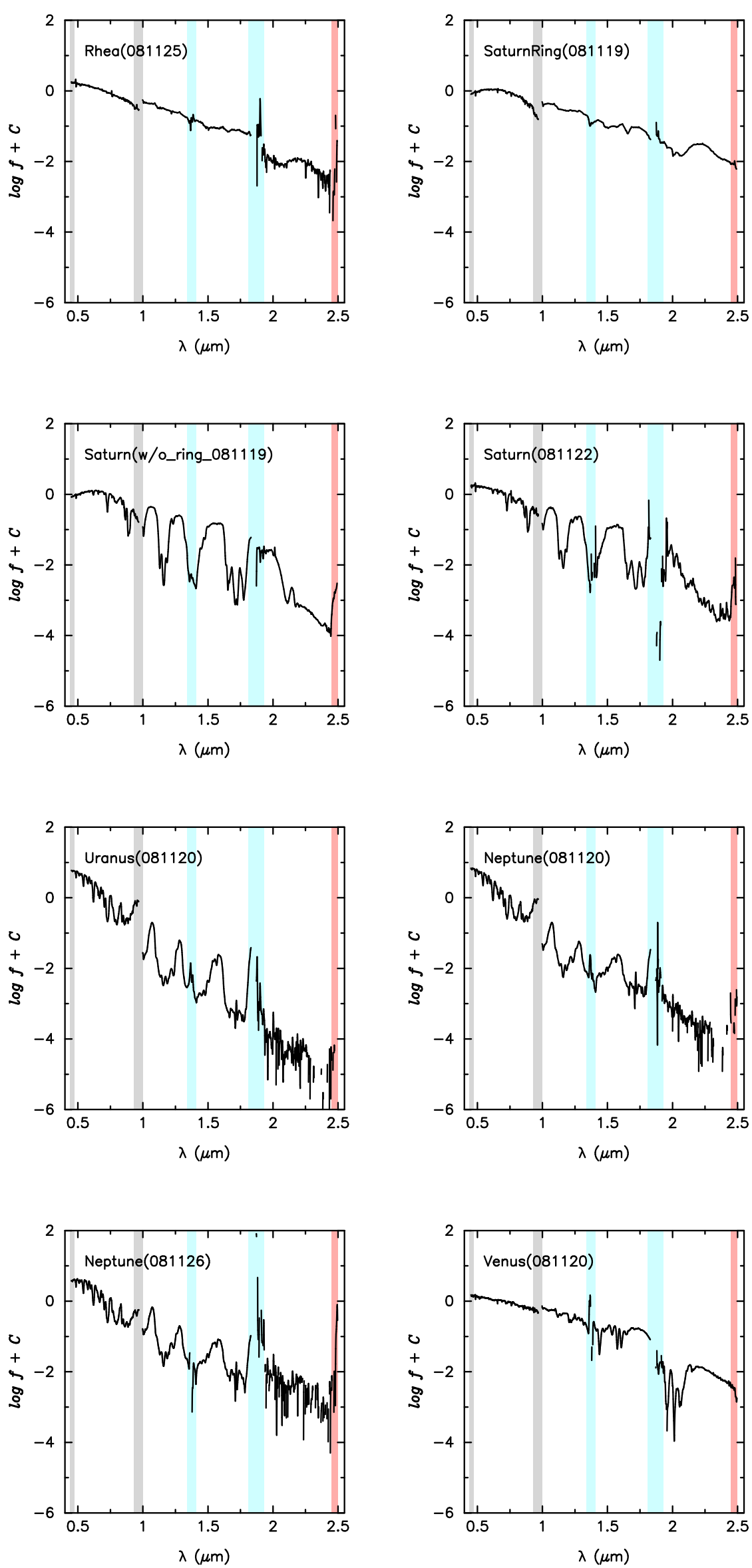

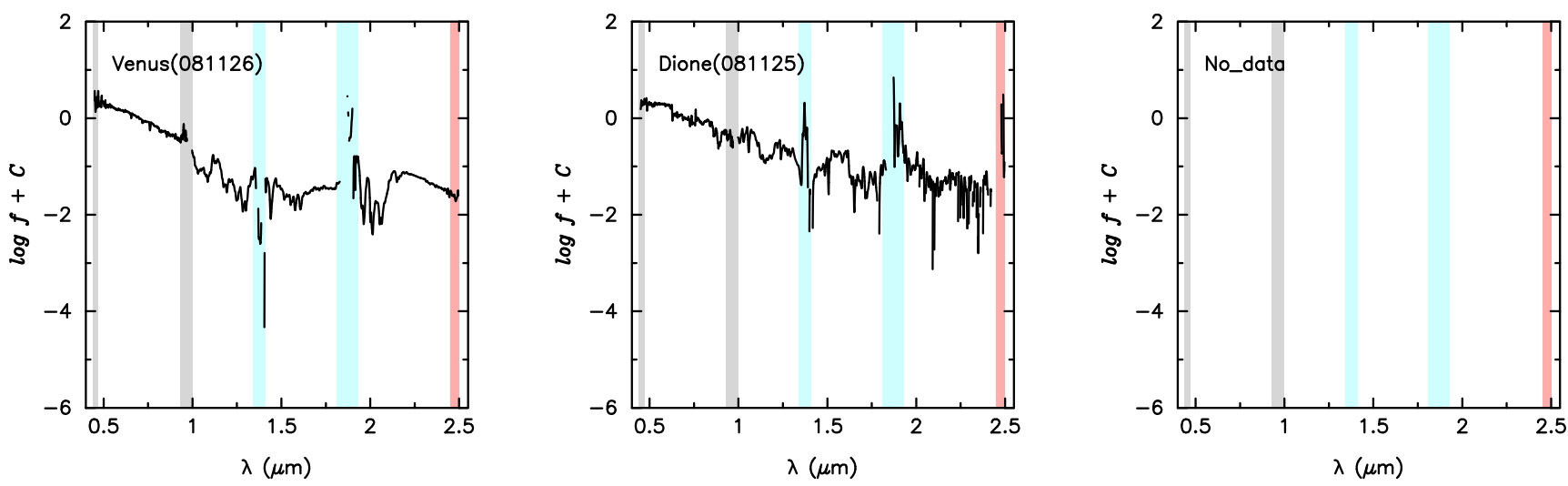

\section{References}

Arnold, L. 2008, Space Sci. Rev., 135, 323

Baudrand, A., Bec-Borsenberger, A., Borsenberger, J., \& Barucci, M. A. 2001, A\&A, 375, 275

Baudrand, A., Bec-Borsenberger, A., \& Borsenberger, J. 2004, A\&A, 423, 381

Belton, M. J. S., Wallace, L., \& Howard, S. 1981, Icarus, 46, 263

Bergstralh, J. T., \& Neff, J. S. 1983, Icarus, 55, 40

Clark, R. N. 1980, Lunar and Planetary Institute, 157

Clark, R. N. 1981, J. Geophys. Res., 86 B4, 2074

Cousins, A. W. J. 1978, Monthly Notes Astron. Soc. South Africa, 37, 8

Delsanti, A., Hainaut, O., Jourdeuil, E., et al. 2004, A\&A, 417, 1145

DeMeo, F. E., Fornasier, S., Barucci, M. A., et al. 2009, A\&A, 493, 283

Fink, U., \& Larson, H. P. 1979, ApJ, 233, 1021

Fink, U., Larson, H. P., Gautier III, T. N., \& Treffers, R. R. 1976, ApJ, 207, L63

Gehrz, R. D., \& Hey, E. P. 1992, Icarus, 100, 162

Grundy, W. M., Buie, M. W., Stansberry, J. A., Spencer, J. R., \& Schmitt, B. 1999, Icarus, 142, 536

Grundy, W. M., Stansberry, J. A., Noll, K. S., et al. 2007, Icarus, 191, 286

Johnson, H. L., \& Morgan, W. W. 1953, ApJ, 117, 313

Karkoschka, E. 1994, Icarus, 111, 174

McCord, T. B. 1981, J. Geophys. Res., 86 B11, 10883

McCord, T. B., \& Westphal, J. A. 1971, ApJ, 168, 141
McCord, T. B., Clark, R. N., \& Huuenin, R. L. 1978, J. Geophys. Res., 83 B11, 5433

Perryman, M. A. C., Lindegren, L., Kovalevsky, J., et al. 1997, A\&A, 323, L49 Pickles, A. J. 1998, PASP, 110, 863

Pieters, C. M. 1986, Rev. of Geophys., 20, 557

Pollack, J. B., Strecker, D. W., Witteborn, F. C., Erickson E. F., \& Baldwin, B. J. 1978a, Icaus, 34, 28

Pollack, J. B., Witteborn, F. C., Erickson, E. F., et al. 1978b, Icarus, 36, 271 Protopapa, S., Boehnhardt, H., Herbst, T. M., et al. 2008, A\&A, 490, 265 Rayner, J. T., Cushing, M. C., \& Vacca, W. D. 2008, ApJ, 678, 1372

Seager, S., Turner, E. L., Schafer, J., \& Ford, E. B. 2005, AstroBio, 5-3, 372 Showman, A. P., Mosqueira, I., \& Head, J. W. 2004, Icarus, 172, 625 Spencer, J. R. 1987, Icarus, 70, 99

Strong, K., Tayor, F. W., Calcutt, S. B., Remedior, J. J., \& Ballard, J. 1993, J. Quant. Spectrosc. Radiat., 50, 363

Tokunaga, A. T., Simons, D. A., \& Vacca, W. D. 2002, PASP, 114, 180 Turnbull, M. C., Traub, W. A., Jucks, K. W., et al. 2006, ApJ, 644, 551 UKIRT Bright Standard Star List, http://www.not.iac.es/instruments/ notcam/ReferenceInfo/bright_stds.html

Vernazza, P., Mothe-Diniz, T., Barucci, M. A., et al. 2005, A\&A, 436, 1113 Watanabe, M., Nakaya, H., Yamauro, T., et al. 2005, PASP, 117, 870

Working Group for Planetary System Nomenclature (WGPSN), http:// planetarynames.wr.usgs.gov/append7.html\#DwarfPlanets

Woolf, N. J., Smith, P. S., Traub, W. A., \& Jucks, K. W. 2002, ApJ, 574, 430 